\title{
An Inquiry-Based Practical Curriculum for Organic Chemistry as Preparation for Industry and Postgraduate Research
}

\author{
Lynne A. Pilcher, ${ }^{a, \star}$, Darren L. Riley ${ }^{a}$, Kgadi C. Mathabathe ${ }^{b}$ and Marietjie Potgieter ${ }^{a}$ \\ ${ }^{a}$ Department of Chemistry, University of Pretoria, South Africa. \\ ${ }^{b}$ Department of Science, Mathematics and Technology Education, University of Pretoria, South Africa.
}

Received 15 June 2015, revised 27 August 2015, accepted 31 August 2015.

\begin{abstract}
This paper describes the development of a new practical curriculum for third-year organic chemistry to replace the recipe-based approach typically used in undergraduate teaching laboratories. The new curriculum consists of an inquiry-based project set in a simulated industrial context preceded by two scaffolding experiments to prepare students for the task. The industrial project requires students to evaluate experimentally three multi-step synthetic routes to a given target based on cost, technical challenge and environmental impact in order to make a recommendation as to which route the 'company' should use to synthesize the compound. The project equips students with technical skills suitable for both postgraduate research and industry, and develops metacognition and understanding through the use of the jig-saw cooperative learning strategy and reflection. The students were found to engage with the practical work at a deep intellectual level, demonstrating that contextualized inquiry-based laboratory teaching afforded an improved quality of learning. In addition, the reported practical curriculum made a difficult subject accessible and even popular, to some measure grew the students' ability in all desired graduate attributes and resulted in the establishment of a professional identity for individual students.
\end{abstract}

KEYWORDS

Inquiry-based, organic chemistry practical training, professional identity, industry-based, metacognition.

\section{Introduction}

Industry today commonly expects graduates to be able to apply theoretical knowledge to practical situations. The truth of the matter is, however, that industry is often faced with the problem of academically strong graduates who are poorly prepared for the practical situations in which they have to perform. As a result new graduates typically find themselves frustrated and struggle to make the transition from university to industry. The biggest shortfall that we have identified in the field of synthetic chemistry is that students are not well equipped to use the available literature and to translate that literature into practice.

Teaching at tertiary level is designed to facilitate preparation of professionals and the laboratory is one of the places where a chemist must operate as a professional person. The aim of laboratory training at university level should be to increase students' ability to act like scientists in the way they solve problems, design investigations, interpret data, troubleshoot methods and set up equipment. ${ }^{1,2}$ Gunstone and Champagne stated that meaningful learning will occur in the laboratory if students are given enough time and are afforded opportunities for inter-personal interactions and reflection. ${ }^{3}$ Domin identified four distinct categories in his taxonomy of laboratory instruction styles, namely exposition, inquiry, discovery and problem-based instruction. ${ }^{1}$ In contrast to the expository style, the other approaches give students more responsibility for and ownership of the activity in which they engage.

Trout et al. refer to inquiry as 'the evidence-based process that scientists engage in to study and propose explanations about * To whom correspondence should be addressed. E-mail: lynne.pilcher@up.ac.za aspects of the natural world' ${ }^{4}$ It involves formulating research questions, proposing methods and strategies for investigation and forming and communicating conclusions. According to Minner et al., for instruction to be classified as inquiry-based it needs to have three aspects: firstly, the presence of science content, secondly, student engagement with science content; and thirdly students must take responsibility for at least one component of the investigation, i.e. question, design, data, conclusion or communication. ${ }^{5}$ Inquiry instruction as a pedagogical approach can be characterized into different categories depending on varying degrees of educator involvement. Buck and co-workers characterized inquiry in the undergraduate laboratory according to the level of student independence, ranging from the confirmation laboratory (level 0 ) where everything is provided to authentic inquiry (level 3) where the problem, procedures, analysis, communication and conclusions are designed by the student. ${ }^{6}$ In guided inquiry (level 1) students look for patterns in data collected via given experimental procedures and in open inquiry (level 2) students design their own experiments to address some general topic. A number of studies have reported the positive impact of some level of inquiry in science instruction on content learning and retention. ${ }^{5}$ Hofstein et al. found that 'students in the inquiry group who had experience in asking questions in the chemistry laboratory outperformed the control grouping in their ability to ask more and better questions'. ' Krystyniak and Heikkinen reported that during an inquiry activity students were less reliant on the instructor than in non-inquiry activities; instead they sought support and guidance regarding experimental procedures from their peers. Furthermore students became confident in their 
understanding of the part for which they were responsible and therefore took on a more active role in the discussions concerning that part of the task. ${ }^{8}$

Metacognition has been defined as exercising control over, being in touch with or reflecting on one's own thinking. ${ }^{9}$ Metacognition is embedded in scientific inquiry because successful inquiry requires a scientist to constantly reflect on and direct his/her thinking towards the desired outcome. The development of metacognitive competencies is a prized outcome of science education because it promotes meaningful learning, autonomy and self-regulation. ${ }^{10}$ The short-term goal for developing thinking and metacognitive skills is to enable students to carry out experiments with understanding. ${ }^{11-12}$ The long-term goal is to develop their problem solving skills in preparation for the world of work. Thus, metacognition is a desired attribute for graduates. ${ }^{13}$ Kipnis and Hofstein arrived at the conclusion that an inquiry-type laboratory assignment that is properly planned and performed carries the potential to provide students with an opportunity to practise and develop metacognitive skills. ${ }^{10}$

The combination of cooperative learning, inquiry instruction and metacognitive instruction creates an environment conducive to encouraging discourse and building of a joint understanding among students. ${ }^{14}$ Given the constraints of time and cost we identified guided inquiry-based instruction with elements of discovery and problem-based learning as the most promising way to develop the problem-solving and planning skills that graduates were lacking.

\section{Context}

This paper reports on the reconceptualization of the practical curriculum for the CMY 384 organic chemistry module at the University of Pretoria. This module is presented to 3rd year students who are enrolled in a Bachelor of Science (BSc) programme with chemistry as a major subject. The BSc programme is run over a three-year period with one quarter (7 weeks, 28 lectures) of each year allocated to organic chemistry lectures. The third year organic syllabus focuses on aromatic and carbonyl chemistry exclusively; the practical curriculum, however, draws on organic chemistry knowledge developed across the entire programme. The practical component of the module comprises $6 \times 6$ h laboratory sessions run over a six-week period, with one practical session per week.

\section{Aims}

The aim of this project was to replace the existing practical component of the CMY 384 module with a curriculum that is holistically designed to prepare students to meet the expectations of their first industrial job or research projects at Honours level. The design specifications for the new curriculum were to include a project in synthetic chemistry that spans several sessions, is inquiry-based, links theory to practice and simulates an industrial context. In addition, it should develop technical skills as well as metacognitive ability.

\section{Design}

The new practical curriculum consists of two preparatory experiments performed in sessions 1 and 2, and an inquirybased industrial project executed during sessions 3 to 6 . The first two sessions (Fig. 1) were used as 'scaffolding' sessions to prepare the students to successfully tackle an inquiry-based project. Students need practice to be able to carry out inquiry-based experiments and should be offered activities that build up to increasingly open and complex levels of inquiry. ${ }^{15}$ Such an approach is not widely implemented in undergraduate laboratory instruction. Since students in the third year organic chemistry module would likely not have experienced an inquiry-based environment, they needed to be systematically equipped with the skills necessary to cope in such an environment. Scaffolding was therefore employed with the first two experiments being dedicated to developing the necessary skills and gradually increasing the level of openness of inquiry with each experiment.

Sessions 3 to 6 comprised a multistep synthetic organic assignment and involved a planning session, two experimental sessions and a presentation session. The project mimics a typical industrial scenario that new graduates might experience when starting out in the workplace. Students are given three different, three-step synthetic routes to the same target molecule to perform, assess and report on.

The first scaffolding experiment (P1) involves the development of extrapolation skills in which students are given a detailed recipe-based procedure in the style of a laboratory notebook record and are then required to extrapolate the reported method and quantities to work with different but related reagents as well as different quantities of reagents. The reaction used is a simple aldol condensation between an aldehyde and a ketone. Four aldehydes and four ketones are provided allowing for up to 16 unique compounds to be prepared. Individual students are each given a different starting reagent combination and have to extrapolate the procedure for their given combination of reagents. They immediately apply this extrapolated procedure as they perform the experiment using their calculated values for the quantities to be used in their experiments.

The key educational aspects at this stage are the development of chemistry mathematical skills and the ability to work independently in a laboratory setting. Mole calculations, introduced in first year theory, are applied in second year practical work; however, by third year a number of students have not mastered these skills. This practical curriculum presents the students with their first exposure to extrapolation and reaction scaling. As students are given different combinations of reagents it is easy to identify those students who are struggling with the concept of stoichiometry and scaling solvent volumes to keep reaction concentration consistent and who require individual instruction. In addition students are put into a situation where they have to work as individuals within the laboratory environment for the first time as they are all doing a different, albeit related, reaction. The development of the ability to work as an individual is seen as a key component of inquiry-based research. Students are encouraged to reason their way through challenges that may arise during execution. This experience promotes confidence and ownership.

The second scaffolding experiment (P2) involves providing

\begin{tabular}{|l|l|l|l|l|l|}
\hline Session $\mathbf{1}$ & Session $\mathbf{2}$ & Session $\mathbf{3}$ & Session $\mathbf{4}$ & Session $\mathbf{5}$ & Session $\mathbf{6}$ \\
\hline Prac $\mathbf{1}$ & Prac 2 & \multicolumn{4}{|l|}{ Prac 3: Inquiry-based Industrial project } \\
\hline P1 & P2 & P3-1 & P3-2 & P3-3 & P3-4 \\
\hline \multicolumn{2}{|c|}{ Scaffolding } & Planning & \multicolumn{2}{|c|}{ Experimentation } & Presentation \\
\hline
\end{tabular}

Figure 1 CMY 384 practical curriculum session breakdown. 
the students with a brief literature style experimental procedure from which the students need to develop their own step-by-step protocol for the experiment. Students are required to perform the experiment using their own protocols after they have been evaluated and corrected by a demonstrator. As with P1, the students are given different combinations of starting reagents and have to extrapolate the procedure, in addition to having to analyse and interpret a literature style method. The educational development at this level builds on that developed in P1; however, it extends to the development of critical interpretation skills. This level of interpretation is not necessarily straightforward as students have at first and second year level been conditioned to expect step-by-step recipe-based synthetic methods and have not given much thought to the rationale for each procedural step. Literature articles typically do not include information that is obvious to someone skilled in the art of synthetic chemistry thus it is important to teach students to make the link.

The inquiry-based project (P3-1 to P3-4) is set in a simulated industrial context. The skill of applying and integrating knowledge to evaluate options and make informed decisions in a relevant context is poorly developed at the third year undergraduate level. ${ }^{16}$ We thought that giving students an opportunity to practise this skill would stimulate the development of this competency. Students are given a brief from a hypothetical company 'Chem-Co Ltd'. The brief details that the company is interested in taking a specific product, 3-phenyl propionate, to market; and that they have identified three potential routes to prepare the product in question. The company asks that teams of chemists assess each route by experimentation in a laboratory environment in terms of three key factors: cost, technical difficulty and environmental impact. Furthermore, in order to keep costs down the company asks that in assessing the routes no more than $2 \mathrm{~g}$ of the final product is produced. Ultimately students have to report their findings as an executive summary in which they make recommendations to the 'board' of Chem-Co Ltd as to which route they feel is best.

The synthetic routes (A, B and C) each consist of a three-step synthesis targeting 3-phenyl propionate (Fig. 2). The routes have been carefully chosen to highlight the three key issues of cost, technical difficulty and environmental impact in such a manner that the question is open-ended with no one clear 'best' route. This project clearly illustrates the concept that there is usually more than one valid route to any compound. The chemistry within the routes is drawn from the organic undergraduate curriculum (years 1 to 3 ): hydrogenation was introduced in the first year, Grignard chemistry in the second year, and at the start of the project students were completing the final chapter of enolate chemistry. Students at this stage were familiar with reaction mechanisms and could use their knowledge to interpret reactions that have not been specifically taught, notably the Knoevenagel reaction (which can be extrapolated from enolate theory) and the particular esterification methods. The routes have comparable time demands, but different levels of complexity.

\section{The Inquiry-based Project}

In this section we provide information on the implementation of the inquiry-based project highlighting organizational and educational aspects. 'Cooperative learning is a student-centred, active-learning approach that uses structured situations in which a fixed small group interacts in a non-competitive manner to accomplish a common goal' ${ }^{17}{ }^{17}$ We decided to use a cooperative learning approach for the following reasons: working through collaboration results in a reduction in cognitive load which facilitates enhanced cognitive and metacognitive activity. ${ }^{18}$ In addition, working through collaboration requires participants to monitor their contribution to the task which obliges participants to explain their ideas and conceptions to others. ${ }^{19} \mathrm{~A}$ laboratory is a social environment where students work independently and in collaboration with peers. Student-student and student-staff interactions play an important role in how students carry out experiments and how they monitor and control their thinking. Students could pool their knowledge and skills to negotiate a common understanding of the task and its demands. They could then select appropriate strategies to perform the task and collectively monitor their thinking and their performance.

\subsection{Session P3-1}

At the start of this session (P3-1), the lecturer in charge gave a ten-minute explanation of the brief and how the project would be run. In order to facilitate cooperative learning and to allow experimental data to be collected for three different routes, i.e. to make the task posed in the brief more manageable, students were divided into teams of chemists using a jigsaw learning group approach. The jigsaw method is a research-based learning technique which was originally developed in 1971 by Elliot

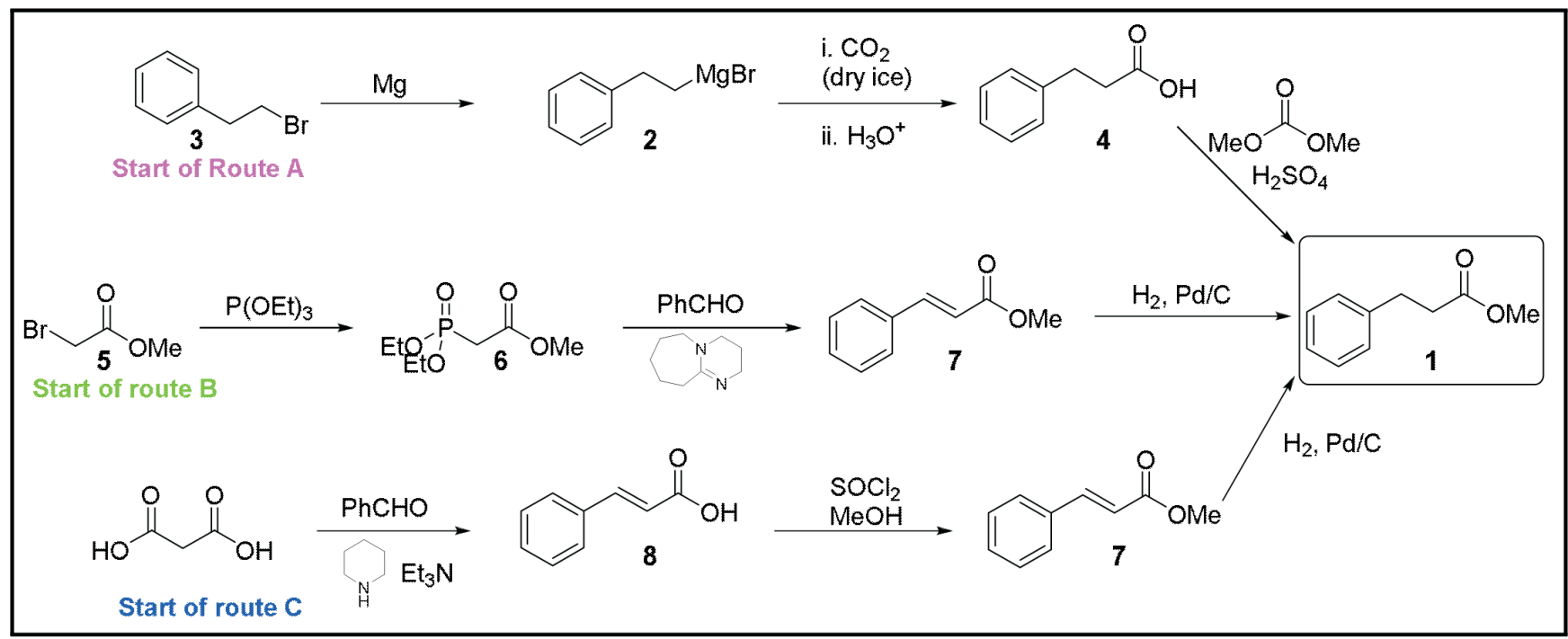

Figure 2 The three multi-step routes (A, B and C) to synthesize methyl 3-phenylpropionate. 
Aronson..$^{20}$ This cooperative learning technique has been studied and used by many researchers and educators in different subjects and at different academic levels. ${ }^{21-23}$ The technique was found effective in increasing positive educational outcomes and cooperation. Similar to a jigsaw puzzle each piece (a student's allocated role) is important for successful completion and full understanding of the task. ${ }^{20}$ Skills such as listening, engagement, empathy, communication and problem-solving are encouraged amongst students. The jigsaw approach involved dividing the students into home groups, each home group comprised three students who were each tasked with assessing one of the synthetic routes (A, B and C) outlined in the brief (Fig. 3).

The group assignments and allocation of routes were only made available to the students upon their arrival for the P3-1 planning session to discourage sourcing of information from students from previous years. The allocation of students was not random but was based on the assessment of the students' abilities during the scaffolding practical sessions (P1 and P2) as well as on peer-friend relationships within the student body. We attempted to place students in groups where they had at least one of: friendship, race or gender in common with the rest of the group members. As far as possible, students were grouped with their friends in home groups to facilitate out of hours preparation of the final report. When this was logistically impossible, students were grouped with a friend in their specialist group to minimize potential discomfort with group work. Within the context of the home group students initially met and were given 10 minutes to discuss their understanding of the brief, their individual roles and expectations of each member of the group, what information they would need to answer the key questions posed in the brief and how they would answer these questions.
The staff member in charge answered any questions that had arisen and the home groups were dissolved. The students were then reassigned to specialist groups. Each specialist group comprised 3-4 students who were all assigned the same synthetic route (Fig. 4). At this stage each specialist group was provided with journal procedures covering the three steps for their synthetic route A, B or C, price lists for the chemicals, MSDS data and a document detailing the twelve principles of green chemistry. The specialist groups were given three hours to translate and extrapolate the journal procedures provided to develop a synthetic strategy that they would use in sessions P3-2 and P3-3. Key aspects that the specialist groups investigated included:reagent quantities to produce approximately $2 \mathrm{~g}$ of 3-phenyl propionate, glassware and equipment, experimental set-up, work-up and purification, costing of chemicals, safety assessment and a time-management plan. In an attempt to add variability and limit copying of results and calculations from year to year or group to group the students are not given the actual reported yield for any of the steps, instead they are provided with suggested yields. The suggested yields are lower than the actual yields reported in the literature which makes them attainable. The use of suggested yields allows the practical coordinator the option to vary the suggestions from year to year or even group to group, adding year-to-year variability in the required calculations. As the specialist groups completed each task, a staff member or demonstrator would check the work to make sure that each group could proceed safely. Thus the specialist group planning session ended with all students having a safe working procedure and correct calculation of quantities of reagents.

The specialist groups were dissolved and students re-formed

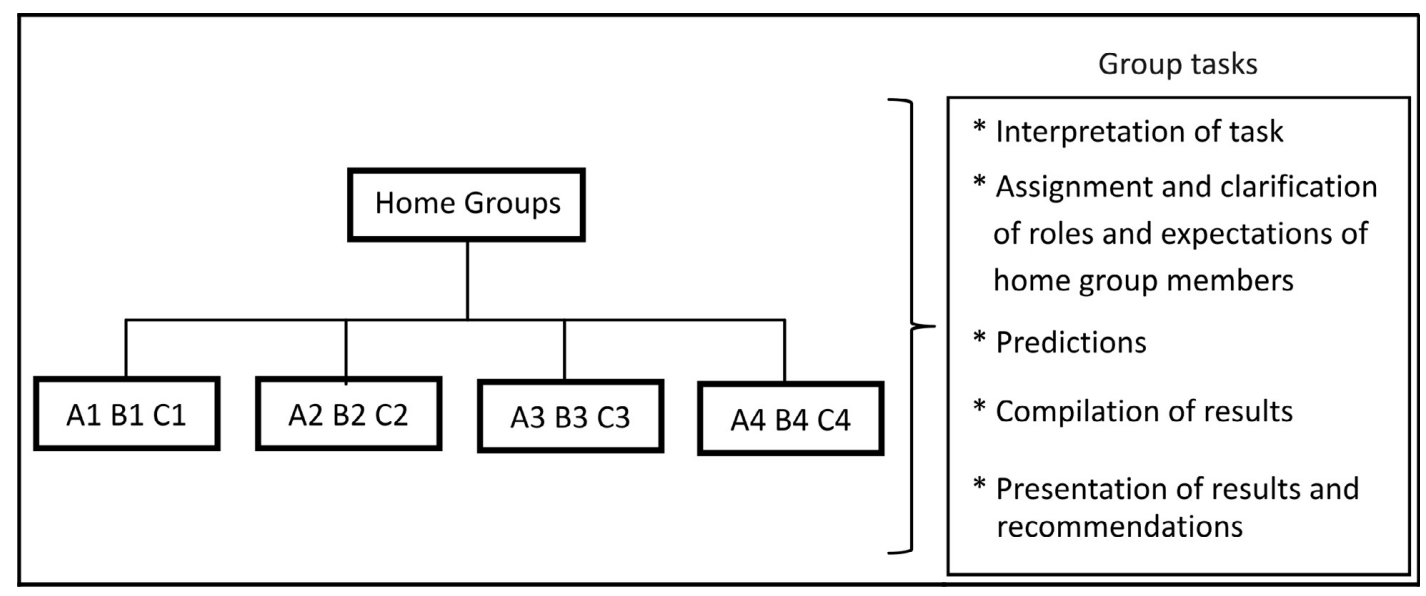

Figure 3 Home group composition and tasks.

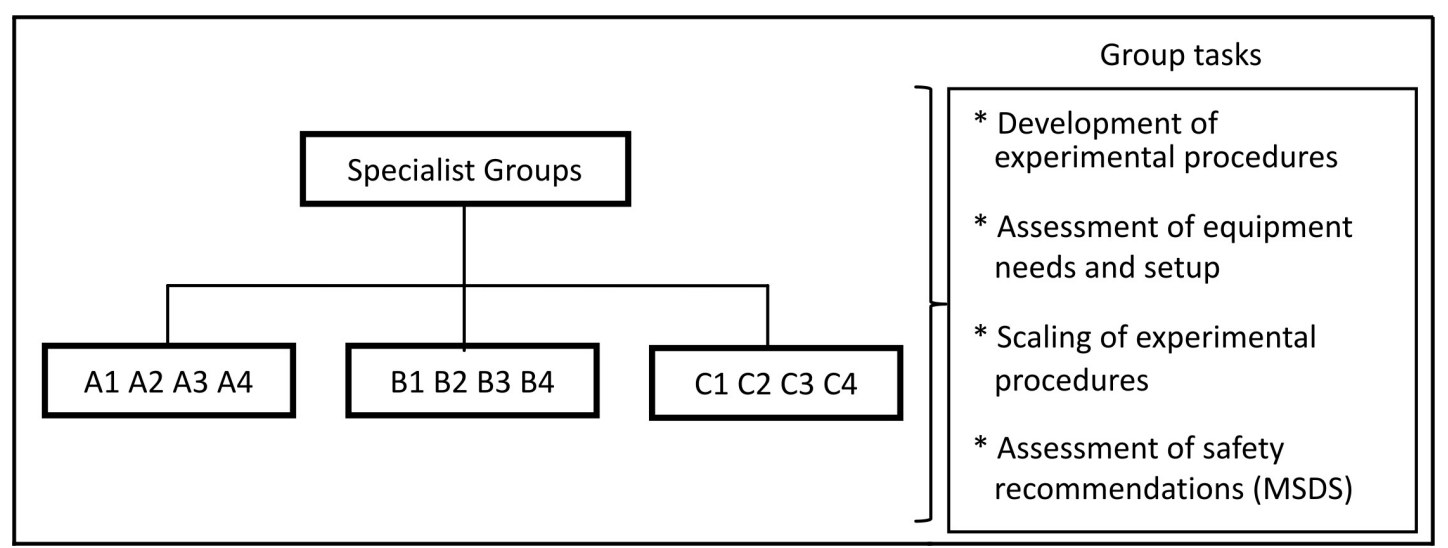

Figure 4 Specialist group composition and tasks. 
their home groups. The individual members of the home group were then given 15 minutes to report back on their findings. At this stage, having assessed the routes, students were tasked with making predictions of how they thought each route would fare against the criteria laid out in the brief. In order for a home group to make a motivated prediction the group needed each member to have processed in full the information particular to his/her route to the extent that it could be evaluated against that of the two other routes. Students were given the remaining time ( 1 to $2 \mathrm{~h}$ ) in session P3-1 to go to the laboratory and prepare their glassware for their first experiment.

\subsection{Sessions P3-2 and P3-3}

The wet laboratory sessions (P3-2 and P3-3) were each 6 hours in length, and it was during this time that the individual students had to assess experimentally their allocated synthetic route. Activities undertaken were those typical of an undergraduate laboratory and included setting up of reactions, reaction monitoring, reaction work-up and purification. Educational aspects that were focused on in addition to development of practical laboratory skills were the development of trouble shooting skills as well as the development of an inquiry-based approach to research. Students were expected to critically examine what they were doing in the laboratory and to formulate their own ideas on how to tackle problems. Academic staff members and demonstrators facilitated this way of thinking through the use of pertinent probing questions in response to students' questions, the objective being that the student should be able to formulate their own answers without the demonstrator having to resort to simply giving an answer. As an additional challenge the students had to fit three synthetic steps into two laboratory sessions one week apart. This required careful time management and that consideration should be given to possible degradation of intermediate products.

\subsection{Session $P 3-4$}

The culmination of the project involved a presentation session (P3-4) where students within their previously allocated home groups were tasked with compiling and presenting their results in a mock board meeting with Chem-Co. Ltd. The home groups were given no indication of how they should structure or present their findings except that it should take the form of a PowerPoint presentation and that every group member should make a contribution. Due to the open-ended nature of the synthetic routes A, B and C, different home groups typically came to different conclusions depending on how much emphasis they put on each of the criteria in the brief as well as their experimental outcomes. As the project is designed to have no one correct answer students were then invited to participate in an open discussion amongst the home groups during which time they could critique each other's recommendations and findings. Finally the session ended with industry-focused feedback from the staff members in charge, highlighting the areas that were not adequately covered or perhaps were not considered by students in the presentations.

\subsection{Development of Metacognitive Skills}

Throughout the inquiry project metacognition was stimulated by way of four questionnaires with reflection prompts which students had to complete before and during the laboratory activity. The questionnaires served as a reminder to students to activate their metacognitive abilities of planning, monitoring, control and evaluation while carrying out the task. Each questionnaire consisted of reflection prompts relevant to different stages of the activity, i.e. during the planning session or during the wet lab activity. The reflection prompts were adapted from Schraw's regulatory checklist which was designed to provide an overarching heuristic that facilitates the regulation of cognition. ${ }^{24}$ Fig. 5 is a diagrammatic representation of when the questionnaires were introduced. It is important to note that these questionnaires, called Reflective Learning Strategy Questionnaires (RLSQs, available as supplementary material), were not designed for assessment purposes but for eliciting productive metacognitive activity.

The Pre-laboratory individual RLSQ encouraged the students to monitor their understanding of the task and to activate prior knowledge after reading the brief which detailed the scope of the task. The students were expected to complete this questionnaire prior to attending planning session P3-1. The pre-laboratory home group questionnaire was divided into two sections: the first was answered before dispersing to specialist groups and prompted them to define what information they needed to bring back from specialist groups; the second was answered after returning from the specialist groups and required an evaluation of information obtained during the specialist group sessions to predict the best route. It also prompted the home group to

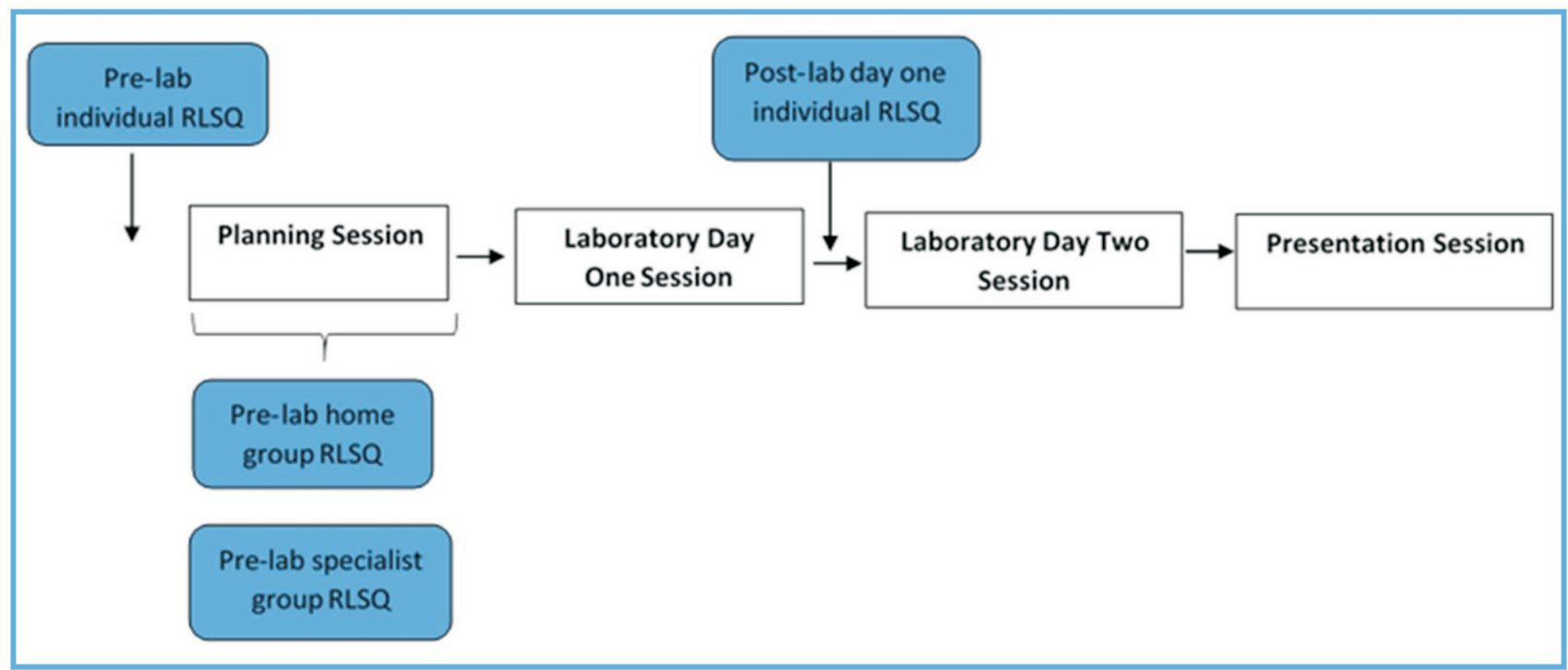

Figure 5 An overview of the four sessions indicating when the four RLSQs were completed. 
plan for the final presentation in session P3-4. The pre-laboratory specialist group questionnaire questions were designed to help the group use their planning time optimally and to evaluate whether the experimental plan and procedure they had just produced during the planning session was complete.

The final RLSQ, the post-laboratory day one RLSQ, was given to the students to complete individually between the two experimental days to help them to evaluate what they would have done differently and how their plans had to change based on their results thus far. Questions like 'Do you have a clear understanding of what you have done and what you still have to do?' and 'If you could repeat today's work, how would you do things differently?' encouraged students to reflect on the events of the day and to evaluate their understanding and progress.

\subsection{Description of Synthetic Routes}

In order to facilitate an open-ended nature to the inquirybased project (P-3) the three routes where designed in such a manner that each route highlighted one of the key aspects raised in the brief (environmental impact, technical difficulty, and cost). A discussion of our evaluation of each route against the three criteria will be made available to bona fida instructors on request.

Route A (Fig. 2) involved the in situ preparation of Grignard reagent 2 from 4 -ethylbromobenzene 3 , the subsequent carboxylic acid 4 was accessed by condensation of 2 with carbon dioxide in the form of dry ice followed by an acidic work-up. Finally the acid was converted into 3-phenyl propionate 1 through treatment with dimethylcarbonate.

Route B involves an Arbuzov reaction of triethylphosphite and $\alpha$-bromo ester 5 followed by a Horner-Wadsworth-Emmons olefination of the resulting phosphonate ester $\mathbf{6}$ with benzaldehyde. Finally catalytic hydrogenation of alkene 7 affords the desired 3-phenyl propionate $\mathbf{1}$.

Route $\mathrm{C}$ involves a Knoevenagel type condensation with benzaldehyde and malonic acid followed by simultaneous $\beta$-decarboxylation to afford acid 8. Esterification using thionyl chloride gives alkene 7 which is hydrogenated under the same conditions as in route B to afford the desired 3-phenyl propionate $\mathbf{1}$.

\subsection{Assignment of Routes}

Students were informally assessed in terms of their experimental ability based upon their in-laboratory performance in experiments 1 and 2 . When the home groups were constituted the student perceived to be weakest was assigned route $C$. The reasoning behind this assignment was twofold, firstly it involves simple experimental set-ups, secondly as the third step is the same as that for route B it affords the student a chance to share the results from their team mate working on route B should their reaction fail or they work too slowly.

\subsection{Assessment}

Individual student assessment in the inquiry-based project (P3) was based on an evaluation of the quality of laboratory notebook records submitted at the end of session 6 , as well as the quality of products of reactions and melting points where applicable. The rubrics developed for the scaffolding experiments were used for these evaluations (see Supplementary material). Marks were also assigned for mechanisms proposed or researched for all synthetic steps and for assignment of ${ }^{1} \mathrm{H}$ and ${ }^{13} \mathrm{C}$ NMR spectra for all products in the student's assigned route. In addition, an assessment of the quality of the slides used by the home group for their final presentation in session 6 contributed a $10 \%$ group mark to the total. Marks were not assigned to their presentation skills because it was felt that students were more likely to engage in the spirit of the exercise when they did not have the added pressure of being assessed for their presentation skills.

\section{Findings}

The results of the inquiry-based industrial project can be divided into two categories: firstly, the results of the students' investigations as revealed by their presentations, and secondly, the attitude and personal development of the students.

\subsection{Students Evaluation of the Three Routes}

Students' communication of their findings in the presentation session was generally of a high standard and in almost all cases the assessment of the three routes was dealt with both critically and logically. Students tended to break down their assessment beyond the three criteria laid out in the brief to include other related aspects such as time demand, waste generated, yields, hazards and number of operations.

The best route, in terms of the criteria laid out in the 'Chem-Co Ltd' brief, chosen by home groups varied. Students tended to favour two routes equally over the third ${ }^{+}$. Typically home groups more focused on environmental impact tended to favour one route, whereas those focused more on cost favoured another. Students regarded new procedures or those that they had had little prior opportunity to practise as technically challenging and unfavourable.

Assessment of the three performance criteria for the synthetic routes was dealt with at a fairly basic level. In terms of costs almost all students were able to price the different routes in terms of the amount of reagents consumed relative to the amount of final product produced. More astute students attempted to look at broader issues which influence cost such as power consumption (ambient temperature $v$ s. refluxed reactions) and time taken (cost of scientists per unit time). We also came across a few students who were breaking the cost assessment down further to include the number of operational steps in the process, and management of by-products and waste. The cost assessment was performed quantitatively based on cost of starting reagents combined with a more qualitative assessment of less easily calculated factors such as power and time consumption.

Although students were easily able to identify areas of technical challenge, they tended to struggle to correlate whether or not the difficulty was because of their own skill levels or because the route and the chemistry itself posed genuine issues. Students tend to be somewhat misled because they were equating technical difficulty with unfamiliarity of a technique regardless of how well the technique worked. As a general rule, groups tended to place less emphasis on technical challenge as a reason for rejecting a particular route.

The final aspect of green chemistry was largely dealt with in a qualitative manner. Students tended to focus on identifying reagents or steps that were not considered to be green in nature, the more observant students tended to consider the by-products and waste produced as well. In the initial implementation of this practical curriculum in 2013 students were mostly unaware of the idea of atom economy and how that relates to greenness. In 2014 we provided students with a synopsis of what atom economy is in addition to the document detailing the twelve principles of green chemistry provided in 2013. Students in the 2014 group

\footnotetext{
${ }^{+}$An explicit description of the findings is avoided in order not to compromise the inquiry aspect and the shelf-life of this experiment for these particular reactions. A document containing detailed analysis of the students' evaluations of the routes will be made available to bona fide researchers and instructors on request.
} 
then tended to emphasize atom economy with the topic being dealt with at some level in almost all presentations. In a few cases students made suggestions of greener approaches that could have been utilized.

\subsection{Student Development}

The scaffolding experiments (P1 and P2) were necessary to ensure students were able to tackle the inquiry-based project (P3). In particular it appeared important that students had developed in two areas: firstly, the ability to work independently, which is not developed during first and second year practical training as the entire class of students would be assigned exactly the same experiments. Secondly, students needed to be capable of interpreting, translating and extrapolating a journal styled experimental procedure as opposed to using a detailed recipe based procedure typically presented at first and second year levels.

When presented with the brief for the inquiry-based project many students expressed apprehension with regard to the difficulty of the task at hand; however, once the realization set in that they were able to successfully tackle the project, students attitudes in almost all cases had a complete turn-around. Evidence of this turn-around was clearly observed in students' overwhelmingly positive assessment of the course. Many students cited the practical component of the module as being their favourite section as demonstrated by the following two comments from an anonymous survey question: What did you like most about the course (module)?

Student 1: 'The practicals were great, it was something different and I learnt a few things that I wouldn't have in a normal practical where everyone synthesised the same compound.'

Student 2: 'I enjoyed the practical components the most as I got to build my confidence with regards to using the instruments...'

Feedback was obtained through conversation with the students, anonymous course evaluation surveys and through feedback from colleagues in other departments. A number of students reported to their biochemistry lecturer that: 1) the last practical project was challenging and very innovative giving exposure to industry realities: problems and corresponding solutions, 2) the new chemistry curriculum was so interesting that they were considering an honours in chemistry rather than honours in biochemistry as initially planned, and 3) the CMY 384 module was the best and most enjoyable module in the BSc degree even though it was one of the most difficult. Students were so excited about the project that they were talking about it outside the confines of the course. In the two years since implementing this practical curriculum we have observed a sharp increase in students' interest in organic chemistry reflected in the increase in the number of students applying to do organic chemistry-based honours projects ${ }^{\ddagger}$.

We were able to observe students' personal development in terms of improved self-confidence, communication skills and troubleshooting abilities. In particular, we were pleasantly surprised by the improvement in the quality of laboratory talk. The interactions of students with staff and demonstrators evolved from that of simply asking for solutions to that of seeking validation for their own ideas which were presented with confidence and were supported by sound arguments. Students acknowledged that this project design improved their understanding of their experimental work:

FWhereas previously less than half our honours students chose to do organic projects, in 2014, 12 out of 13 students and in 201510 out of 12 students chose organic chemistry honours projects.
'I think people would get a lot better result if we actually thought about the experiment but if we are not really forced to do that in the way that we were in this activity we probably won't.' (Quote from a post-course interview)

In their reasoning students demonstrated an improved ability to think at three levels: molecular, macroscopic and symbolic and to switch seamlessly between these thinking levels as indicated by this student comment given in a post-course interview:

\section{'...you were just given a recipe you don't really know what you are doing ... and you don't [know] what's really happening molecular, .... But now on this third year level and with this experiment you gain an appreciation of what real life is gonna be like. So you have to figure out for yourself.'}

Another fundamental and rather exciting observation that we made having adopted the new inquiry-based model combined with a simulation of an industry environment is that students began to develop a professional identity wherein they started to believe that they were no longer simply students learning about chemistry but actual chemists capable of doing research. This is illustrated by the following student comment in a response to a survey:

'The experiments done in this course gave us as students more insight in how the actual work might work in the real world and it was very educational to develop the methods ourselves instead of just reading a manual.'

Interactions in the laboratory between students and lecturers became an equal exchange between professionals to formulate ideas and critique them. This transformation was especially evident during the presentations of the teams of chemists to the 'board' of Chem-Co. Students took ownership of both the process and the outcomes. This observation was in sharp contrast to the past where, presumably because of a limited job market specific to chemistry, our students did not have a sense of belonging to a professional community. Development of a professional identity gave the students confidence and a story to tell as they could see how their studies equipped them to function in a relevant working situation.

\section{Discussion and Conclusions}

It is the desire of most institutions of higher learning to produce graduates who are critical thinkers, who are confident in the work environment; who can function independently; who are lifelong learners; who can generate new ideas, resolve problems, create products and come up with solutions that are valuable to society. ${ }^{13,25}$ After completion of this inquiry-based industrial project, our students had developed in each of the attributes listed by the University of Pretoria (Fig. 6).

This programme addressed each one of the graduate attributes. Our approach of giving students individual tasks meant that they had to learn to work independently. As a result of independent achievement, their self-confidence grew. We introduced an inquiry-based approach and this stimulated the intellectual curiosity of our students. By modelling an industrial environment the students gained understanding of economic and industrial realities, not formally part of the syllabus. The use of the jigsaw method required students to work in different teams. Their role in each team and the contribution they had to make developed interpersonal skills as well as accountability to the group. Our students learnt to apply their theoretical knowledge to develop experimental methods and to solve problems in the laboratory. Requiring students to consider the environmental ef- 


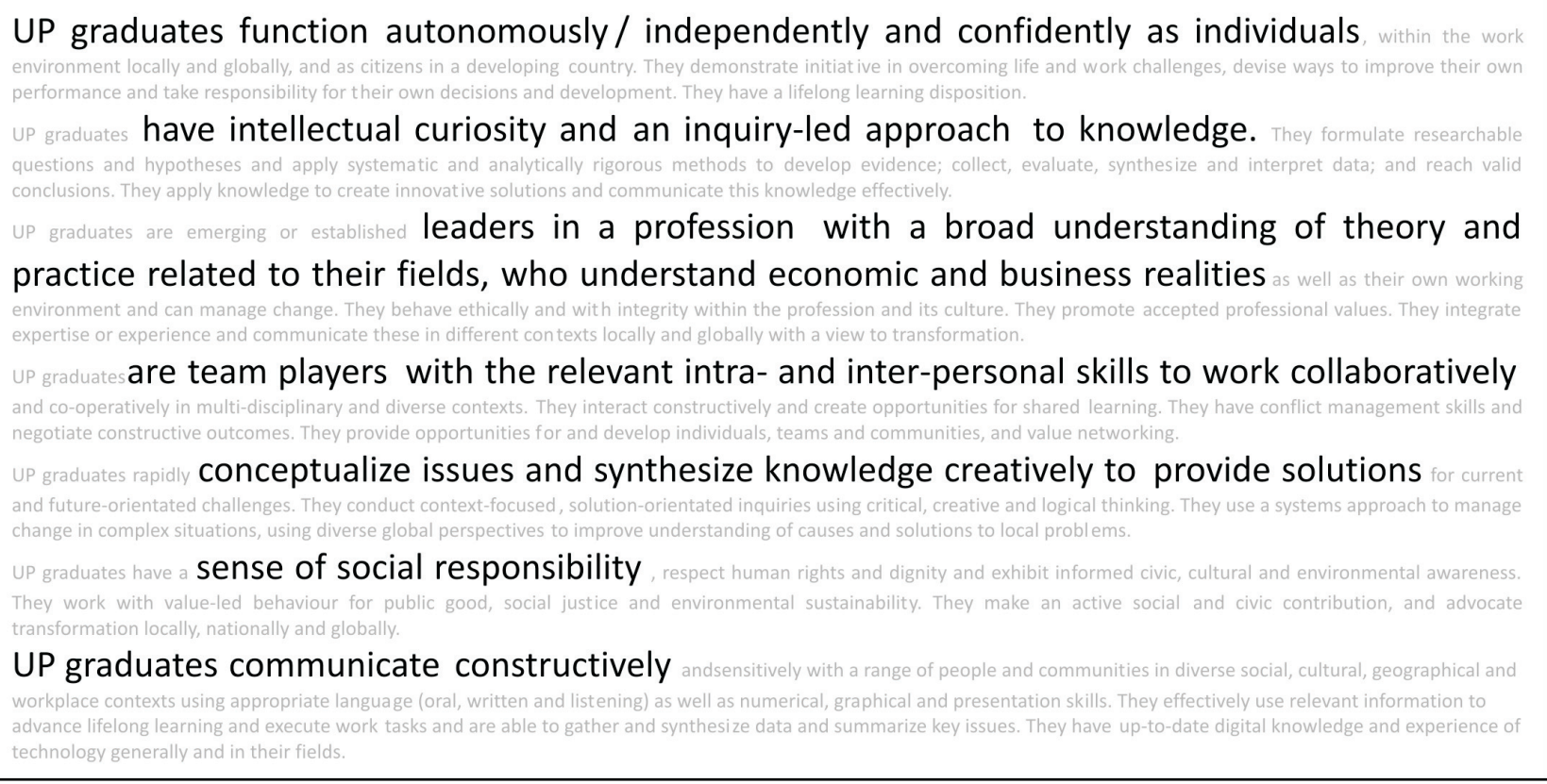

Figure 6 Statement of graduate attributes for the University of Pretoria.

fects of their synthetic routes fostered a sense of social responsibility. Our students developed a sense of ownership of their work which enabled them to communicate with clarity and authority when presenting their findings.

In addition, the graduate attributes that industry expects from organic chemists were also addressed. Our students' ability to plan and design experimental procedures based on abbreviated journal-like descriptions and relevant theory was developed. Students were able to estimate the initial scale of a reaction based on the final downstream product quantity required. They gained experience in interpreting MSDS data and all safety aspects associated with chemicals. Manipulative skills and skills of record keeping and data handling were practised. Our students grew in their ability to think at the symbolic, macroscopic and molecular levels for interpretation of observations and experimental results and for problem solving.

Experimental chemistry, with its associated chemical hazards and cost of reagents ${ }^{\S}$, is generally believed to be closed to the introduction of authentic inquiry-based learning at undergraduate level. The design of this project kept the inquiry aspect to an evaluation of a pre-planned set of experimental routes. Thus the chemistry was already tested, the safety aspects well considered, time constraints were accounted for and the chemicals were available. This guided-inquiry approach encompassed the benefits of inquiry while being well targeted to the experience level of our students and the level of expectation for a new graduate.

Three aspects of this curriculum design that are of paramount importance to the chemistry educator deserve further comment, namely the expected shelf-life of the open-endedness of the industry-based project, the range of practical skills covered by the curriculum and the transferability of the design to contexts with a three-hour duration of practical sessions.

To maintain the open-ended inquiry aspect of the project, it is important that following a number of years of implementation a so-called 'correct answer' does not become known. We antici-

sIn the South African context, long waiting times for delivery after placing an order, also represent a problem. pate that over time students may be informed by students from previous years how the routes will fare against one another. However, students will still have to perform the experiments to compare yields and cost. Furthermore, in planning their experiments quantities can be varied by varying the expected yields for the reactions and even the target quantity to be produced. This inquiry-design is not specific to the chosen chemistry and could conceivably be applied to any set of synthetic routes to a common target molecule that were relevant to the theory curriculum.

The benefits of the allocation of four laboratory sessions to one practical activity, with reallocation of two experimental sessions to planning and reporting, far outweighed the loss in terms of skills development of exposing students to a different experiment in each session. As expected, providing students with enough opportunity for interactions with peers and instructors and opportunities for reflection and metacognitive activity, resulted in enhanced understanding of what they were expected to do and why they were doing it.,12 Although a student performed only one of the synthetic routes they were exposed to the technical challenges associated with all three routes in their home groups where results were discussed to reach a joint conclusion. The combination of this cooperative learning, inquiry instruction and metacognitive prompting created an environment conducive to encouraging discussion and joint building of understanding amongst students. ${ }^{14}$

While the practical slot in the timetable for the CMY 384 module is 6 hours, many institutions timetable 3 hour sessions for their practical training with more sessions being allocated to the practical programme. The reactions used in this project are either complete within 2 hours or must be left overnight. It should therefore be possible to adjust the project to work in a 3 hour context with reactions being set up and quenched in one session or left 'overnight' until the next session and alternate sessions being dedicated to reaction work up and product isolation. In such a context eight 3 hour sessions would be required: two for planning and preparation for the first reaction, five for experimentation and one for reporting. 
To conclude, we return to perhaps the most gratifying experience during the implementation of this new curriculum, namely to witness how many of the students developed a sense of professional identity while engaged in the industrial project. Professional identity formation is key to success as 'identity is an underlying motivation for learning' ${ }^{26}$ When students perceive an activity to be meaningful they are intrinsically motivated to master the required skills and concepts at a much deeper level than when driven by external gains such as grades. Unlike students in professional training such as health practitioners and engineers with a clear career track following completion of their studies, science students are slow to develop this identity. In his study on the epistemic development of organic chemists Bhattacharyya made three recommendations to support students in their development of a professional identity namely, the inclusion of professionally relevant elements in their training, opportunities to experience the roles performed by practitioners, and opportunities to determine the problems of the profession and search for solutions through feedback and reflection. ${ }^{26}$ The practical curriculum reported here meets all three of these recommendations. Students who have benefited from this experience are expected to transfer successfully to industry or postgraduate research because not only have they acquired the appropriate knowledge and skills they also see themselves as professional chemists equipped to make a meaningful contribution.

\section{Supporting Information Available}

In order not to compromise the inquiry aspect of the design for this particular set of reactions, the supplementary material will not be generally accessible on-line, but will be made available to bona fide researchers and instructors on request from the authors.

1. Chemistry design principles for each route and detailed student evaluations of each route.

2. 'Chem-Co Ltd.' Brief

3. RLSQs

4. Rubrics for evaluating laboratory notebooks and samples.

5. Documentation for specialist groups A, B and C (Literature procedures, costs of reagents, notes on Green Chemistry, ${ }^{1} \mathrm{H}$ and ${ }^{13} \mathrm{C}$ NMR spectra)

6. Laboratory manager notes

7. Demonstrator notes (provides the essence of the training the demonstrators need to run the practical as well as tips for anticipating reaction problems.)

8. Academic leader notes.

\section{Acknowledgements}

We thank Bonolo Moruri for testing all reactions described in this paper prior to implementation of the curriculum. This work was supported by an Education Innovation grant from the University of Pretoria.

\section{References}

1 D.S. Domin, A Review of laboratory instruction styles, J. Chem. Ed., 1999, 76, 543-547.

2 R. Lippmann Kung and C. Linder, Metacognitive activity in the physics student laboratory: Is increased metacognition necessarily better?, Metacog. Learn., 2007, 2, 41-56.

3 R.F. Gunstone and A.B. Champagne, Promoting conceptual change in the laboratory, in The Student Laboratory and the Science Curriculum, (E. Hegarty-Hazel, ed.), Routledge, London, 1990, pp. 159-182.

4 L. Trout, C. Lee, R. Moog and D. Rickey, Inquiry Learning: What Is It? How Do You Do It?, retrieved on 27 May 2015 from https:// www.acs.org/content/dam/acsorg/education/policies/hsstandards/chapter-4.pdf.

5 D.D. Minner, A.J. Levy and J. Century, Inquiry-based science instruction - What is it and does it matter? Results from a research synthesis years 1984 to 2002, J. Res. Sci. Teach., 2010, 47, 474-496.

6 L.B. Buck, S. L. Bretz and M.H. Towns, Characterizing the level of inquiry in the undergraduate laboratory, J. College. Sci. Teach., 2008, 52-58.

7 A. Hofstein, O. Navon, M. Kipnis and R. Mamlok-Naaman, Developing students' ability to ask more and better questions resulting from inquiry-type chemistry laboratories, J. Res. Sci. Teach., 2005, 42, 791-806.

8 R.A. Krystyniak and H.W. Heikkinen, Analysis of verbal interactions during an extended, open-inquiry general chemistry laboratory investigation, J. Res. Sci. Teach., 2007, 44, 1160-1186.

9 J.H. Flavell, Metacognition and cognitive monitoring a new era of cognitive-developmental inquiry, Am. Psych., 1979, 34, 906-911.

10 M. Kipnis and A. Hofstein, The inquiry laboratory as a source for development of metacognitive skills, Int. J. Sci. Math. Educ., 2008, 6, 601-627.

11 A. Hofstein and V.N. Lunetta, The laboratory in science education: foundation for the 21st century, Sci. Educ., 2004, 88, 28-54.

12 R.F. Gunstone, Reconstructing theory from practical experience, in Practical Science, (B.E. Woolnough, ed.), Open University Press, Milton Keynes, 1991, pp. 67-77.

13 J.A. Slabbert, D.M. de Kock and A. Hattingh, The Brave 'New' World of Education: Creating a Unique Professionalism, Juta and Company, Cape Town, South Africa, 2009.

14 J.M. Kittleson and S.A. Southerland, The role of discourse in group knowledge construction: a case study of engineering students, J. Res. Sci. Teach., 2004, 41, 267-293.

15 R.A. Bell, L. Smetana and I. Binns, Simplifying Inquiry Instruction, retrieved on 9 April 2013 from www.nsta.org/publications/news/story.aspx?id $=50983,2005$.

16 S. Cullipher, H. Sevian and V. Talanquer, Reasoning about benefits, costs, and risks of chemical substances: mapping different levels of sophistication, Chem. Educ. Res. Pract., 2015, 16, 377-392.

17 S. Sandi-Urena, M.M. Cooper and R.H. Stevens, Effect of cooperative problem-based lab instruction on metacognition and problemsolving skills, Int. J. Chem. Educ., 2012, 89, 700-706.

18 D. Whitebread, P. Coltman, D.P. Pasternak, C. Sangster, V. Grau, S. Bingham, Q. Almeqdad and D. Demetriou, The development of two observational tools for assessing metacognition and selfregulated learning in young children, Metacog. Learn., 2009, 4, 63-85.

19 T. Iiskala, M. Vaurus and E. Lehtinen, Socially shared metacognition in peer-learning? Hel. J. Psych., 2004, 1, 147-178.

20 Aronson, E., Jigsaw Classroom. Retrieved on 27 May 2015 from http:// www.jigsaw.org/index.html, 2000.

21 E. Aronson, N. Blaney, C. Stephin, J. Sikes and M. Snapp, The Jigsaw Classroom, Sage Publishing Company, Beverly Hills, California, 1978.

22 Y.J. Dori and O. Herscovitz, Question-posing capability as an alternative evaluation method: analysis of an environmental case study. J. Res. Sci. Teach., 1999, 36, 411-430.

23 Q. Mengduo and J. Xiaoling, Jigsaw Strategy as a cooperative learning technique: focusing on the language learners. Chi. J. App. Linguist., 2010, 33, 113-125.

24 G. Schraw, Promoting general metacognitive awareness. Inst. Sci., 1998, 26, 113-125.

25 University of Pretoria draft statements of graduate attributes (2012)

26 G. Bhattacharyya, Who am I? What am I doing here? Professional identity and the epistemic development of organic chemists, Chem. Educ. Res. Pract., 2008, 9, 84-92. 